Acta Crystallographica Section D

Biological

Crystallography

ISSN 0907-4449

\section{Cheng-Guo Wu, ${ }^{\text {a }}$ Shu-Chun Cheng, ${ }^{\text {b }}$ Shiang-Chuan Chen, Juo-Yan Li, ${ }^{a}$ Yi-Hsuan Fang, Yau-Hung Chen ${ }^{b}$ and Chi-Yuan Chou $^{\text {a* }}$}

${ }^{a}$ Department of Life Sciences and Institute of Genome Sciences, National Yang-Ming

University, Taipei 112, Taiwan, and

${ }^{\mathbf{b}}$ Department of Chemistry, Tamkang University, Tamsui 251, Taiwan

Correspondence e-mail: cychou@ym.edu.tw

\title{
Mechanism for controlling the monomer-dimer conversion of SARS coronavirus main protease
}

The Severe acute respiratory syndrome coronavirus (SARS$\mathrm{CoV})$ main protease $\left(\mathrm{M}^{\text {pro }}\right)$ cleaves two virion polyproteins (pp1a and pp1ab); this essential process represents an attractive target for the development of anti-SARS drugs. The functional unit of $\mathrm{M}^{\text {pro }}$ is a homodimer and each subunit contains a His41/Cys145 catalytic dyad. Large amounts of biochemical and structural information are available on $\mathrm{M}^{\text {pro }}$; nevertheless, the mechanism by which monomeric $\mathrm{M}^{\text {pro }}$ is converted into a dimer during maturation still remains poorly understood. Previous studies have suggested that a C-terminal residue, Arg298, interacts with Ser123 of the other monomer in the dimer, and mutation of Arg298 results in a monomeric structure with a collapsed substrate-binding pocket. Interestingly, the R298A mutant of $\mathrm{M}^{\text {pro }}$ shows a reversible substrateinduced dimerization that is essential for catalysis. Here, the conformational change that occurs during substrate-induced dimerization is delineated by X-ray crystallography. A dimer with a mutual orientation of the monomers that differs from that of the wild-type protease is present in the asymmetric unit. The presence of a complete substrate-binding pocket and oxyanion hole in both protomers suggests that they are both catalytically active, while the two domain IIIs show minor reorganization. This structural information offers valuable insights into the molecular mechanism associated with substrate-induced dimerization and has important implications with respect to the maturation of the enzyme.

\section{Introduction}

Coronaviruses (CoVs) belong to the order Nidovirales, which are enveloped positive-stranded RNA viruses with a large genome of about $30 \mathrm{~kb}$ (Gorbalenya et al., 2006). They include important pathogens of humans and other animals (Weiss \& Navas-Martin, 2005). In late 2002, a novel CoV causing severe acute respiratory syndrome (SARS) with a $15 \%$ fatality rate emerged and spread to three continents in six months (World Health Organization; http://www.who.int/csr/sars/country/ 2003_08_15/en/). In the following few years, the discovery of two further species of human CoVs, NL-63 and HCoV-HKU1, as well as SARS-CoV-like strains in bats, confirmed the great diversity of CoVs (van der Hoek et al., 2004; Lau et al., 2005; Li et al., 2005; Woo et al., 2005). In September 2012, the World Health Organization was informed of several cases of acute respiratory syndrome with renal failure owing to infection with a novel $\mathrm{CoV}$ in the Middle East. The possible global health implications are still under critical evaluation. Nevertheless, this re-emphasizes the possibility of the future re-
Received 22 October 2012

Accepted 13 January 2013

PDB Reference: SARS-CoV $M^{\text {pro }}$, R298A mutant, 4hi3
(C) 2013 International Union of Crystallography Printed in Singapore - all rights reserved 
emergence of SARS or a related disease. Therefore, studies to aid our understanding of these viruses and the development of novel antiviral inhibitors are both urgent and necessary.

The coronavirus nonstructural polyproteins (pp1a and pp1ab) are cleaved by two kinds of viral cysteine proteases: a main protease $\left(\mathrm{M}^{\mathrm{pro}}\right.$ or $3 \mathrm{CL}^{\text {pro }}$; EC 3.4.22.69) and a papainlike protease (EC 3.4.22.46; Snijder et al., 2003). This process is considered to be a suitable antiviral target because cleavage is required for viral maturation (Wu et al., 2006; Chou et al., 2008; Zhu et al., 2011). $\mathrm{M}^{\text {pro }}$ cleaves the polyproteins at 11 sites that contain the canonical L-Q- $\downarrow-(\mathrm{A} / \mathrm{S} / \mathrm{N})$ sequence (Hegyi \& Ziebuhr, 2002). Catalysis by $\mathbf{M}^{\text {pro }}$ has been studied extensively over the years using both kinetic and mutagenesis approaches (Anand et al., 2003; Chou et al., 2004; Hsu, Kuo et al., 2005; Lin et al., 2008; Shi et al., 2008; Chen et al., 2008; Hu et al., 2009; Cheng et al., 2010). Structural information is also available on $\mathrm{M}^{\text {pro }}$ from SARS CoV and many other CoVs (Anand et al., 2002; Yang et al., 2003; Xue et al., 2008; Zhao et al., 2008). The structure of the coronaviral $\mathrm{M}^{\text {pro }}$ consists of three domains and the catalytic dyad His/Cys is located at the interface between domains I and II. The first two domains have an antiparallel $\beta$-barrel structure that forms a folding scaffold similar to other viral chymotrypsin-like proteases (Anand et al., 2002, 2003). Domain III is a five-helix fold that contributes to the dimerization of $\mathbf{M}^{\text {pro }}$ (Anand et al., 2002, 2003). There is a very long loop (residues 176-200) between domains II and III. Recent studies have suggested that foldon unfolding of SARS-CoV $\mathrm{M}^{\text {pro }}$ domain III alone is able to mediate the interconversion between the monomer and a three-dimensional domainswapped dimer under physiological conditions (Kang et al., 2012); nevertheless, how the two domain IIIs meet and remain together until $\alpha$-helix swapping takes place is still unknown.

Mature $\mathbf{M}^{\text {pro }}$ is a stable homodimer in which the two subunits are arranged perpendicularly to each other (Yang et al., 2003). Mutation or deletion of the $\mathrm{N}$-finger (the first seven residues) and the C-terminus (residues 298-306) can lead to a

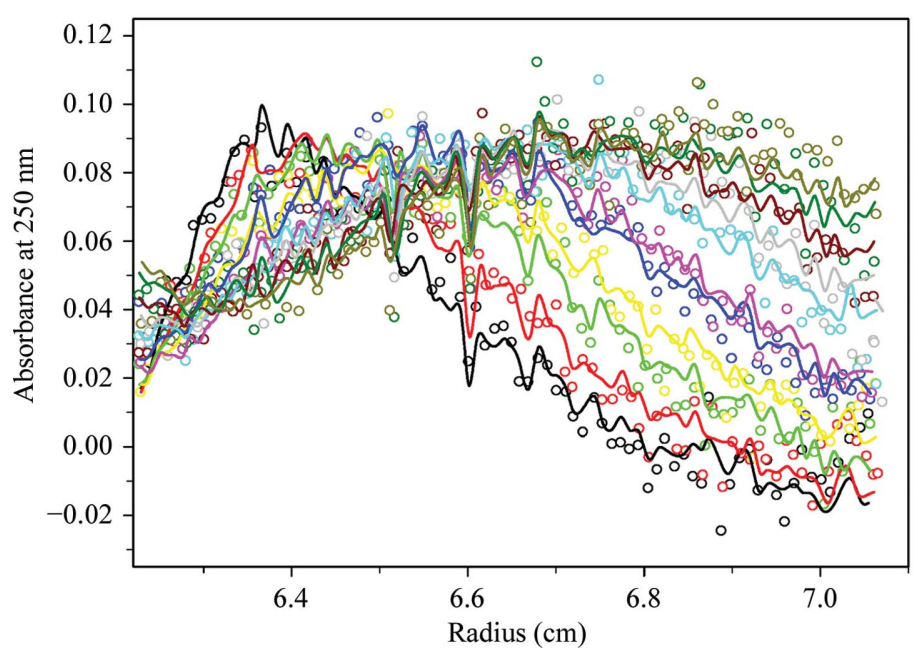

(a)

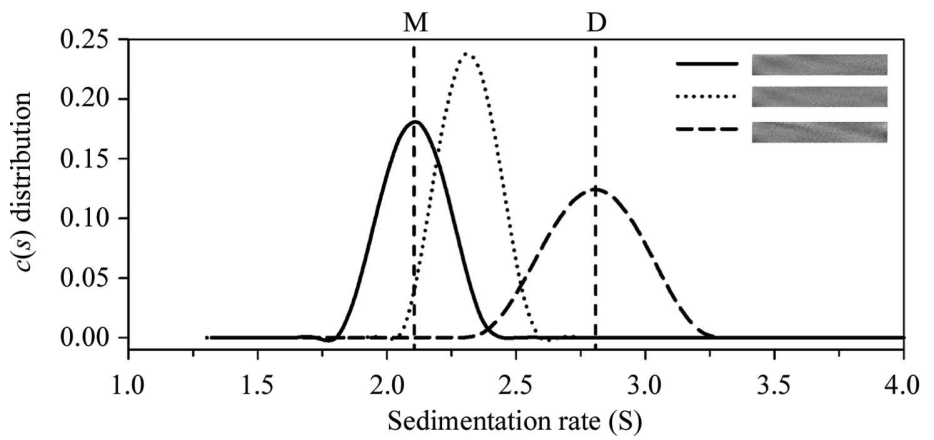

(c)

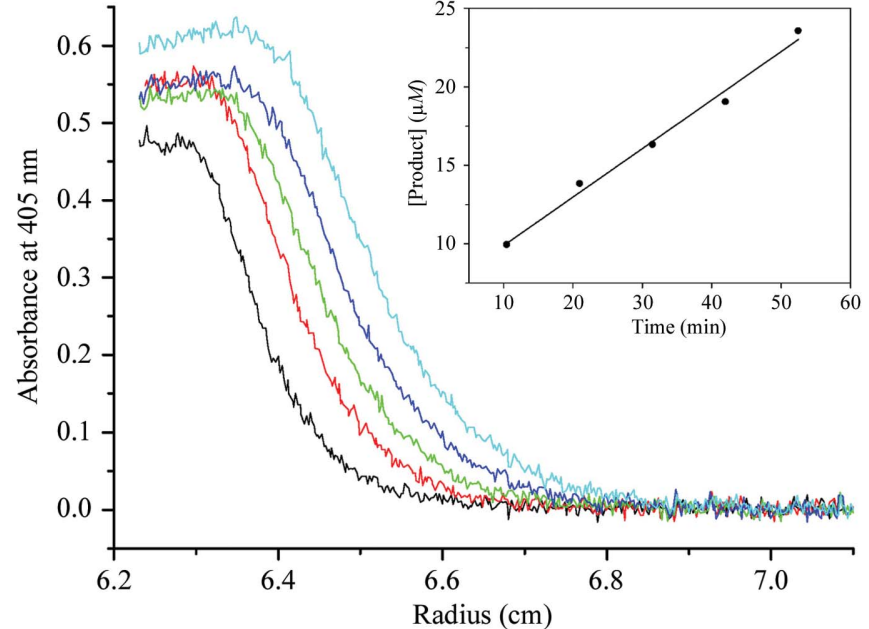

(b)

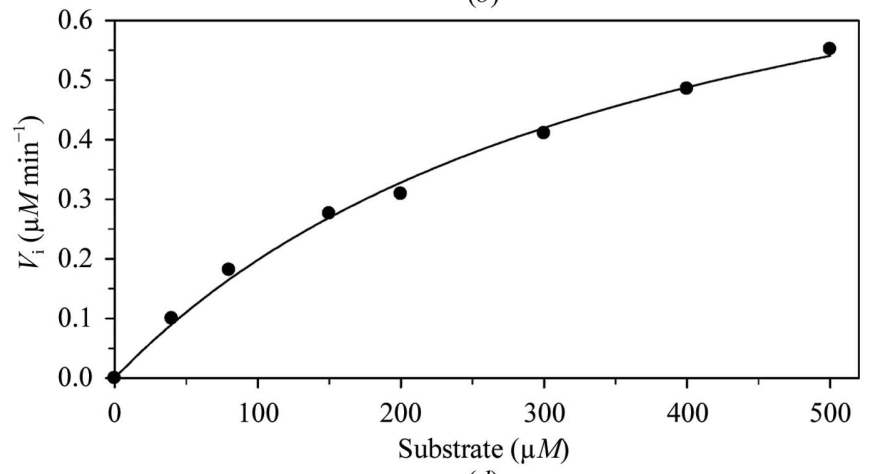

$(d)$

Figure 1

AEC pattern of the R298A mutant of SARS-CoV M ${ }^{\text {pro }}$. The amount of protein used was $15 \mu \mathrm{l}\left(1 \mathrm{mg} \mathrm{ml}^{-1}\right)$ and the total volume of the cell was $330 \mu \mathrm{l}$. (a) A typical trace of the absorbance at $250 \mathrm{~nm}$ of the R298A mutant during an experiment at a substrate concentration of $200 \mu M$. The symbols represent experimental data and the lines are the results fitted to the Lamm equation using the SEDFIT program (Chou et al., 2011; Schuck, 2000). The best-fit distribution result is shown by dashed lines in $(c)$. (b) The absorbance at $405 \mathrm{~nm}$ tracing the released product (pNA) after the first hour of the same experiment. The time interval between two successive spectra, from black to cyan, is $10 \mathrm{~min}$. The inset plot shows the product at different times. The line indicates the best-fit result for the initial velocity calculation. (c) The continuous $c(s)$ distributions of the $\mathrm{M}^{\text {pro }} \mathrm{R} 298 \mathrm{~A}$ mutant from the best-fit analysis of the $250 \mathrm{~nm}$ results. The distributions in $10 \mathrm{~m} M$ phosphate buffer $\mathrm{pH} 7.6$ are shown by solid lines, while those in the presence of peptide substrate at 40 and $200 \mu M$ are shown as dotted and dashed lines, respectively. The two straight dashed lines indicate the positions of the monomer (M) and dimer (D). The residual bitmaps of the raw data and the best-fit results are shown as insets. (d) Plot of the initial velocities (from $405 \mathrm{~nm}$ results) versus substrate concentration. The line represents the best-fit results according to the Michaelis-Menten equation. 
monomeric $\mathrm{M}^{\text {pro }}$ with little enzyme activity (Yang et al., 2003; Chou et al., 2004; Hsu, Chang et al., 2005). $\mathrm{M}^{\text {pro }}$ containing additional $\mathrm{N}$ - and $\mathrm{C}$-terminal segments of the polyprotein undergoes autoprocessing to yield the mature protease in vitro (Hsu, Kuo et al., 2005). Inactive as a monomer, the binding of the peptide substrate or of an N-terminally and/or C-teminally elongated $\mathrm{M}^{\text {pro }}$ molecule is able to induce dimerization of $\mathrm{M}^{\text {pro }}$, allowing catalysis (Cheng et al., 2010; Chen et al., 2010; Li et al., 2010). The effect of substrate-induced dimerization is reversible and can be blocked by the mutation of a key residue, Glu166, which is responsible for the binding to Ser1 of the other protomer and is one of the residues recognizing Gln P1 of the substrate (Anand et al., 2002; Yang et al., 2003; Cheng et al., 2010). In crystal structures of monomeric mutants of SARS-CoV M $\mathrm{M}^{\text {pro }}$, such as R298A or G11A, the oxyanion loop (Ser139-Cys145) is converted to a short $310^{-}$helix; this leads to complete collapse of the oxyanion hole, resulting in enzyme inactivation (Shi et al., 2008; Hu et al., 2009).

Despite the availability of a large amount of biochemical and structural information on $\mathrm{M}^{\text {pro }}$, the mechanism by which monomeric $\mathrm{M}^{\text {pro }}$ is converted to a dimer during the maturation process is currently poorly understood. Here, we report the crystal structure of the SARS-CoV M $^{\text {pro }}$ R298A mutant in the presence of peptide substrate. The structure reveals a functional dimeric form but with a minor change in the relative orientation of the two domain IIIs. Detailed exploration of this structure provides a better and more detailed understanding of the mechanisms that control the dimerization of coronaviral $\mathrm{M}^{\text {pro }}$.

\section{Materials and methods}

\subsection{Protein expression and purification}

The R298A mutant of SARS-CoV $\mathrm{M}^{\text {pro }}$ inserted into the pET-28a(+) vector (Cheng et al., 2010) was expressed in Escherichia coli BL21 (DE3) cells. Cultures were grown in LB medium at $310 \mathrm{~K}$ for $4 \mathrm{~h}$ and were then induced with $0.4 \mathrm{~m} M$ isopropyl $\beta$-D-1-thiogalactopyranoside; this was followed by overnight incubation at $293 \mathrm{~K}$. After centrifugation at $6000 \mathrm{~g}$ at $277 \mathrm{~K}$ for $10 \mathrm{~min}$, the cell pellets were resuspended in lysis buffer ( $20 \mathrm{~m} M$ Tris $\mathrm{pH} 8.5,250 \mathrm{~m} M \mathrm{NaCl}, 5 \%$ glycerol, $0.2 \%$ Triton X-100, $2 \mathrm{~m} M \beta$-mercaptoethanol) and lysed by sonication. The crude extract was then centrifuged at $12000 \mathrm{~g}$ at $277 \mathrm{~K}$ for $25 \mathrm{~min}$ to remove the insoluble pellet. The supernatant was incubated with $1 \mathrm{ml} \mathrm{Ni}-\mathrm{NTA}$ beads (Qiagen, Hilden, Germany) at $277 \mathrm{~K}$ for $1 \mathrm{~h}$ and loaded into an empty column. After flowthrough and washing with washing buffer (20 $\mathrm{m} M$ Tris pH 8.5, $250 \mathrm{mM} \mathrm{NaCl}, 8 \mathrm{~m} M$ imidazole, $2 \mathrm{mM}$ $\beta$-mercaptoethanol), the protein was eluted with elution buffer (20 $\mathrm{m} M$ Tris pH 8.5, $30 \mathrm{mM} \mathrm{NaCl}, 150 \mathrm{~m} M$ imidazole, $2 \mathrm{~m} M \beta$-mercaptoethanol). The resulting protein fraction was then loaded onto an S-300 gel-filtration column (GE Healthcare) equilibrated with running buffer $(20 \mathrm{~m} M$ Tris $\mathrm{pH} 8.5$, $100 \mathrm{~m} M \mathrm{NaCl}, 2 \mathrm{~m} M$ dithiothreitol). The purity of the collected fractions was analyzed by SDS-PAGE. Fractions containing the $\mathrm{M}^{\text {pro }}$ protein were pooled and concentrated to
Table 1

Summary of crystallographic information for $\mathrm{M}^{\text {pro }} \mathrm{R} 298 \mathrm{~A}(\mathrm{pH} 8.0$ ).

Values in parentheses are for the highest resolution shell.

\begin{tabular}{|c|c|}
\hline Space group & $P 1$ \\
\hline Unit-cell parameters $\left(\AA{ }^{\circ}\right)$ & $\begin{array}{c}a=55.0, b=59.4, c=59.8 \\
\alpha=71.3, \beta=73.4, \gamma=72.3\end{array}$ \\
\hline Resolution $(\AA)$ & $30-2.09(2.20-2.09)$ \\
\hline$R_{\text {merge }} \dagger(\%)$ & $3.5(22.0)$ \\
\hline$\langle I / \sigma(I)\rangle$ & $21.5(3.2)$ \\
\hline Completeness (\%) & $94.7(82.6)$ \\
\hline No. of reflections & $35837(4672)$ \\
\hline Multiplicity & $2.2(2.0)$ \\
\hline$R$ factor $\ddagger(\%)$ & 19.0 \\
\hline Free $R$ factor $\$(\%)$ & 24.1 \\
\hline R.m.s.d., bond lengths $(\AA)$ & 0.009 \\
\hline R.m.s.d., bond angles $\left({ }^{\circ}\right)$ & 1.3 \\
\hline
\end{tabular}

$\dagger R_{\text {merge }}=\sum_{h k l} \sum_{i}\left|I_{i}(h k l)-\langle I(h k l)\rangle\right| / \sum_{h k l} \sum_{i} I_{i}(h k l)$, where $I_{i}(h k l)$ is the integrated intensity of a given reflection and $\langle I(h k l)\rangle$ is the mean intensity of multiple corresponding symmetry-related reflections. $\ddagger R=\sum_{h k l}|| F_{\text {obs }}|-| F_{\text {calc }}|| / \sum_{h k l}\left|F_{\text {obs }}\right|$, where $F_{\text {obs }}$ and $F_{\text {calc }}$ are the observed and calculated structure factors, respectively. $\&$ The free $R$ factor is the $R$ factor calculated using a random $5 \%$ of data that were excluded from the refinement.

$30 \mathrm{mg} \mathrm{ml}^{-1}$ using an Amicon Ultra-4 $30 \mathrm{kDa}$ centrifugal filter (Millipore). The typical yield of protein was $5-10 \mathrm{mg}$ per litre of cell culture.

\subsection{Active enzyme centrifugation}

Active enzyme centrifugation (AEC) can be used to observe quaternary-structural changes and catalytic activity simultaneously (Chou et al., 2011). The analytical ultracentrifugation experiments were performed with an XL-A analytical ultracentrifuge (Beckman, Fullerton, California, USA) using an An-50 Ti rotor (Cheng et al., 2010). A commercially available double-sector Vinogard-type bandforming centrepiece (Beckman, Fullerton, California, USA) was used for AEC (Chou et al., 2011; Chou \& Tong, 2011). Briefly, $15 \mu \mathrm{l}$ of the R298A mutant of $\mathrm{M}^{\text {pro }}\left(1 \mathrm{mg} \mathrm{ml}^{-1}\right)$ was added to the small well above the sample sector. After the cell had been assembled, $330 \mu \mathrm{l}$ peptide substrate (TSAVLQ-pNA from GL Biochem, Shanghai, People's Republic of China) dissolved in $\mathrm{D}_{2} \mathrm{O}$ at 0,40 or $200 \mu M$ was loaded into the bulksample sector space. Centrifugation was then carried out at $42000 \mathrm{rev} \mathrm{min}^{-1}$. Absorbance at $250 \mathrm{~nm}$ was chosen to allow detection of the protein band, while absorbance at $405 \mathrm{~nm}$ was used to monitor the catalytic release of the product, pNA. The spectrum was recorded continuously using a time interval of $600 \mathrm{~s}$ per scan and a step size of $0.003 \mathrm{~cm}$. A typical trace of the $250 \mathrm{~nm}$ spectral results is shown in Fig. 1(a). The data set was then fitted to a continuous $c(s)$ distribution model using the SEDFIT program (Schuck, 2000; Brown \& Schuck, 2006). The signals at $405 \mathrm{~nm}$ were used to calculate the initial velocities and were then fitted to the Michaelis-Menten equation, from which the kinetic parameters $K_{\mathrm{m}}$ and $k_{\text {cat }}$ were determined (Chou et al., 2011).

\subsection{Protein crystallization}

Crystals of the R298A mutant were obtained at $295 \mathrm{~K}$ by the sitting-drop vapour-diffusion method. The protein solution was set up at $15 \mathrm{mg} \mathrm{ml}^{-1}$ and included $1 \mathrm{~m} M$ TSAVLQ-pNA. 
The reservoir solution consisted of $0.1 M$ Tris $\mathrm{pH}$ 8.0, $30 \%(w / v)$ PEG 300, 5\%(w/v) PEG 1000. Large but poorly diffracting crystals appeared in $3 \mathrm{~d}$ and were used for microseeding. Single crystals of cubic shape and with dimensions of $0.2-0.3 \mathrm{~mm}$ were obtained in less than a week. All crystals were cryoprotected in reservoir solution with $1 \mathrm{~m} M$ TSAVLQpNA and were then flash-cooled in liquid nitrogen. Crystal soaking in $2 \mathrm{~m} M$ TSAVLQ-pNA failed to improve the electron density of the substrate. Soaking at higher peptide concentrations was impossible owing to low solubility. size-distribution model, it was obvious that the R298A mutant was monomeric $(2.1 \mathrm{~S})$ in the absence of substrate (Fig. 1c, solid lines), whereas it was dimeric (2.8 S) at a substrate concentration of $200 \mu M$ (Fig. 1c, dashed lines). Somewhat surprisingly, at a substrate concentration of $40 \mu M$ the major species $(2.3 \mathrm{~S}$ ) of the R298A mutant was located between the monomer and the dimer (Fig. 1c, dotted lines). According to previous studies, these results suggested that the R298A mutant is a rapid self-association protein, similar to wild-type $\mathrm{M}^{\text {pro }}$ (Cheng et al., 2010). These observations using AEC

\subsection{Data collection, structure determination and refinement}

$\mathrm{X}$-ray diffraction data were collected at $100 \mathrm{~K}$ on SPXF beamline $13 \mathrm{~B} 1$ at the National Synchrotron Radiation Research Center (Taiwan) using an ADSC Quantum-315r CCD (X-ray wavelength $0.976 \AA$ ). The diffraction images were processed and scaled using the $H K L-2000$ package (Otwinowski \& Minor, 1997). The crystal belonged to space group $P 1$, with unit-cell parameters $a=55.0, b=59.4, c=59.8 \AA$, $\alpha=71.3, \beta=73.4, \gamma=72.3^{\circ}$. There are two $\mathrm{M}^{\text {pro }}$ molecules in the asymmetric unit. The structure was solved by the molecular-replacement method with the program Phaser (McCoy et al., 2007) using the structure of wild-type $\mathrm{M}^{\text {pro }}$ (PDB entry 1uk4) as the search model (Yang et al., 2003). Manual rebuilding of the structure model was performed with Coot (Emsley \& Cowtan, 2004). Structure refinement was carried out using the program REFMAC (Murshudov et $a l ., 2011)$. Data-processing and refinement statistics are summarized in Table 1 . The crystal structure has been deposited in the Protein Data Bank (PDB entry 4hi3).

\section{Results and discussion}

\subsection{Substrate-induced dimerization of SARS-CoV $\mathbf{M}^{\text {pro }}$}

To explore the influence of substrate binding on the dimerization of $\mathrm{M}^{\text {pro }}$, we performed AEC experiments on the R298A mutant of $\mathrm{M}^{\text {pro }}$ with or without the peptide substrate. Figs. 1(a) and 1(b) show typical absorbance traces at 250 and $405 \mathrm{~nm}$ of the R298A mutant during the experiment at a substrate concentration of $200 \mu \mathrm{M}$. After fitting the $250 \mathrm{~nm}$ signals to the continuous (a)

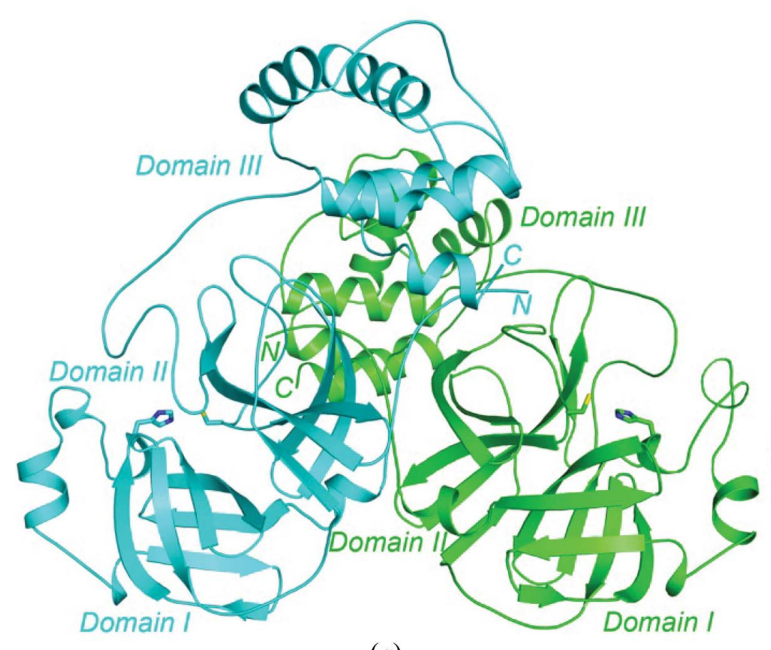

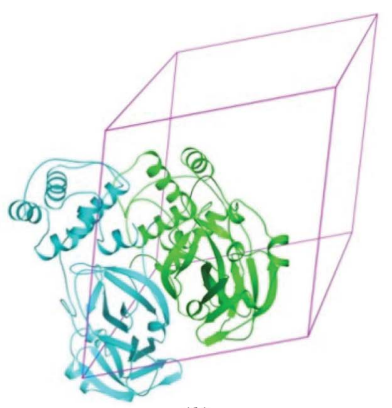

(b)

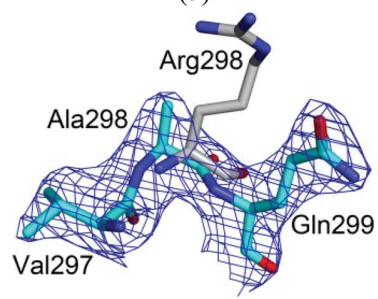

(c)

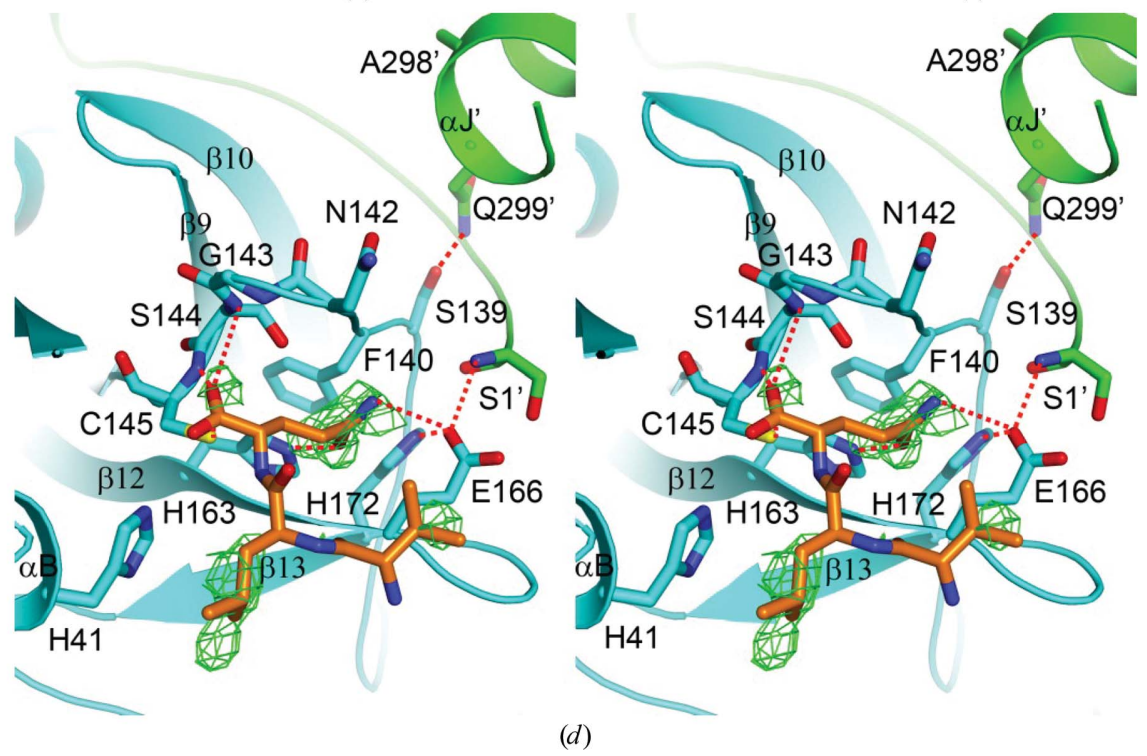

Figure 2

Dimeric structure of the R298A mutant of SARS-CoV M ${ }^{\mathrm{pro}}$. (a) The overall structure of the dimeric $M^{\text {pro }}$ R298A mutant. The two protomers are coloured cyan and green, respectively. (b) Crystal packing of the R298A mutant. Two molecules form a biological dimer in the unit cell. (c) Final $2 F_{\mathrm{o}}-F_{\mathrm{c}}$ electron density contoured at $1.0 \sigma$ for residues $297-299$ of the R298A mutant. The Arg298 residue in wild-type Mpro is also shown (grey). (d) Schematic drawing in stereoview showing the detailed interactions at the active site of the R298A mutant. The residual electron density around the active site is contoured at 2.5 (green mesh) and the C atoms of the modelled P3-P1 (Val-LeuGln) residues are coloured orange. Hydrogen-bonding interactions are indicated by red dashed lines. All structure figures were produced with PyMOL (http://www.pymol.org). 
confirmed the substrate-induced dimerization of the R298A mutant. Moreover, after calculating the initial velocities from signals at $405 \mathrm{~nm}$ and then fitting them to the MichaelisMenten equation (inset in Figs. $1 b$ and $1 d$ ), an apparent $K_{\mathrm{m}}$ of $380 \mu M$ and an apparent $k_{\text {cat }}$ of $0.012 \mathrm{~s}^{-1}$ were calculated. These values are very close to those for wild-type $\mathrm{M}^{\text {pro }}$ from AEC analysis (Chou et al., 2011). This confirmed that the dimeric R298A protein is functionally the same as that of wildtype $\mathrm{M}^{\text {pro }}$. However, based on the crystal structure of the monomeric R298A mutant (Shi et al., 2008), the transition from monomer to dimer should be impossible because of the dramatic rotation of domain III and the formation of a short $3_{10}$-helix from an active-site loop; these changes result in the catalytic machinery being frozen in a collapsed state. Furthermore, recent studies suggested that the R298E mutant maintained $\mathrm{N}$-terminal autocleavage activity comparable to that of wild-type $\mathrm{M}^{\text {pro }}$, although it did cause complete dimer dissociation and disruption of trans-cleavage activity (Chen et al., 2010). This indicated that N-terminal autocleavage of $\mathrm{M}^{\text {pro }}$ is not dependent on a 'mature' dimeric protease, but on an

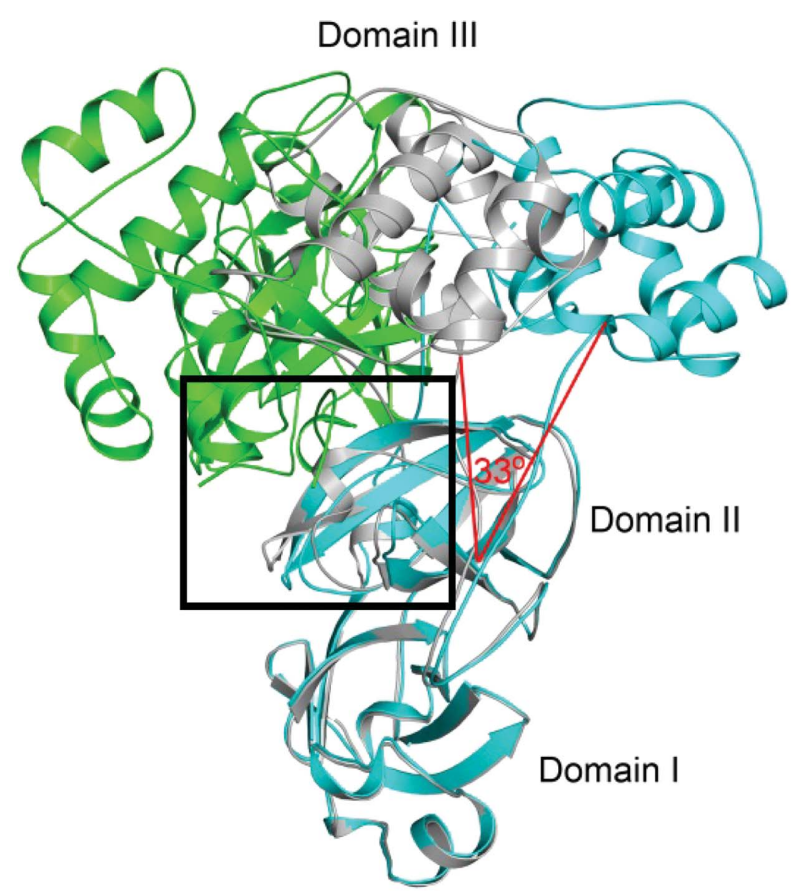

(a)

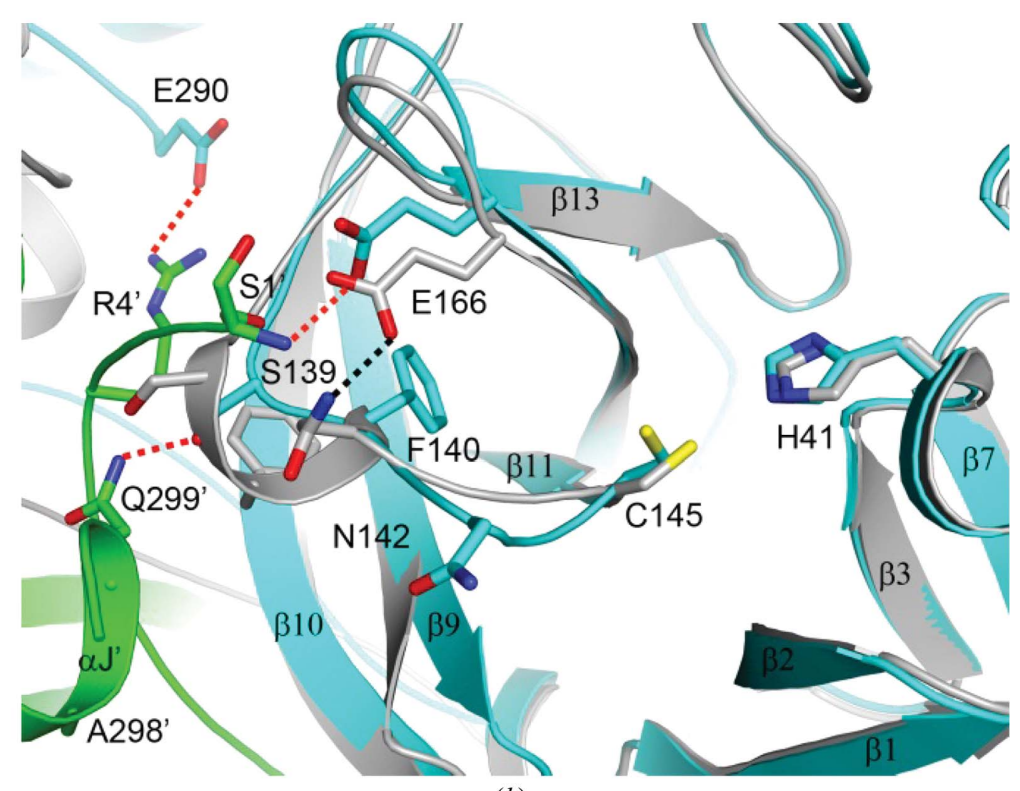

(b)

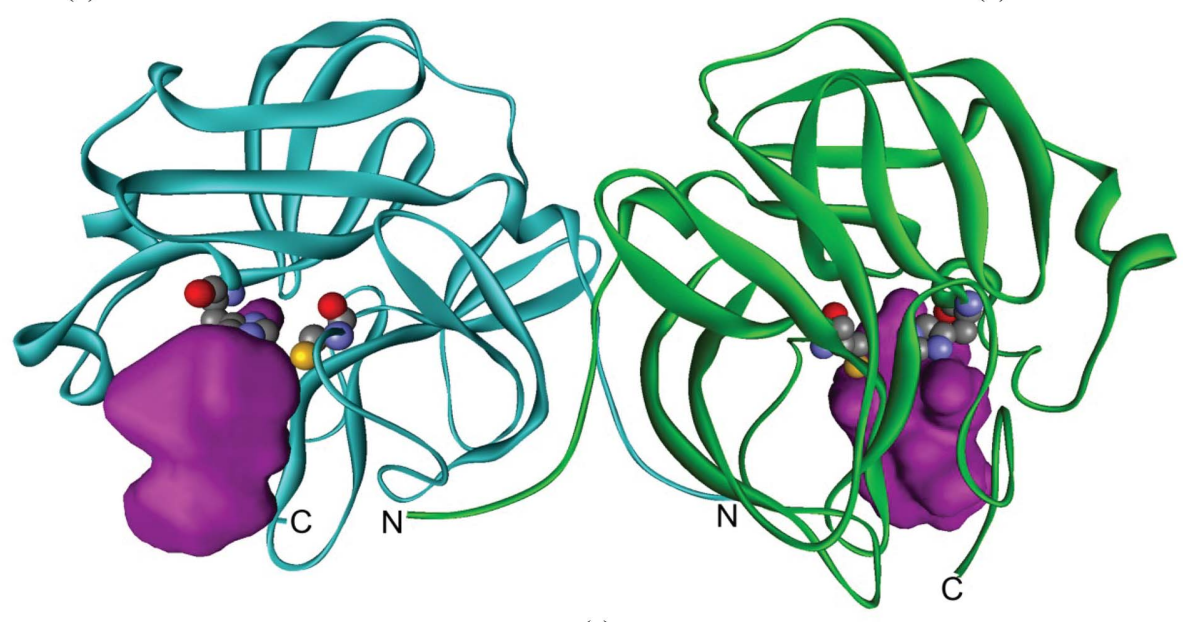

(c)

\section{Figure 3}

Comparisons with the monomeric structure of the R298A mutant of $\mathrm{M}^{\mathrm{pro}}$. (a) Overlay of the current structure of the R298A dimer (in cyan and green) with that of the R298A monomer (grey; Shi et al., 2008). The orientation of domain III shows a $33^{\circ}$ change compared with that in the monomer. The red lines represent the positions of residues 193-200 in the two structures. The region in the box is enlarged in $(b)$. (b) Detailed interactions at the dimer interface of the R298A mutant. The red dashed lines indicate hydrogen bonds associated with the dimer, while black dashed lines indicate hydrogen bonds in the monomer. $(c)$ Substrate-binding cavities mapped onto the current structure. Both protomers show a large and deep cavity (magenta surface representation) near the catalytic dyad, which is essentially the same as that in wild-type $\mathrm{M}^{\text {pro }}$ at $\mathrm{pH} 7.6$, although wild-type $\mathrm{M}^{\text {pro }}$ at $\mathrm{pH} 6.0$ shows one active protomer and one inactive protomer (Yang et al., 2003). The cavity was calculated using DS Modeling 1.7 (Accelrys) and was drawn using Discovery Studio Visualizer 2.5 (Accelrys). 
'intermediate' or 'loose' dimer (Chen et al., 2010). On the other hand, we have expressed and purified an R298A mutant with an $\mathrm{N}$-terminal extension similar to that of the R298E mutant (Chen et al., 2010). However, the mutant lost its transcleavage activity (less than $0.8 \%$ ) and failed to form a dimer even in the presence of peptide substrates (Supplementary Fig. $1^{1}$ ). Efforts to crystallize the $\mathrm{N}$-terminally extended R298A mutant were also unsuccessful. To delineate the mechanism for the conversion of $\mathrm{M}^{\text {pro }}$ from an inactive monomer to a functional dimer, we next determined the crystal structure of the dimeric R298A mutant at a resolution of $2.09 \AA$ (Table 1). It is important to note that we used an R298A mutant with an authentic $\mathrm{N}$-terminus and a C-terminus with eight extra residues $\left(\mathrm{LEH}_{6}\right)$ for convenience in purification. According to previous studies (Chen et al., 2010), in the presence of peptide substrates the structure should be more like a mature dimer with trans-cleavage activity, not a premature dimer with an $\mathrm{N}$-terminal extension.

\subsection{Overall structure of the R298A mutant of SARS-CoV $M^{\text {pro }}$ in the presence of peptide substrate}

The original goal of our experiment was to determine the binding modes of peptide substrates to the R298A mutant. We therefore included high concentrations of peptide substrate ( $1 \mathrm{~m} M$; threefold higher than the $K_{\mathrm{m}}$ ) in the cocrystallization conditions. The refined atomic model agreed well with the crystallographic data and the expected bond angles and bond lengths (Table 1). About $91 \%$ of the residues are in the most favoured region of the Ramachandran plot; none are in the disallowed region.

Consistent with our expectations and the AEC results, two essentially identical monomers of the R298A mutant exist in the asymmetric unit, with an r.m.s.d. of $1.1 \AA$ between their $\mathrm{C}^{\alpha}$ atoms (Figs. $2 a$ and $2 b$ ). There is a minor change in orientation between the two copies of domain III and therefore the r.m.s.d. value decreases to $0.28 \AA$ when domain III is excluded. The overall structure of the dimeric R298A mutant is similar to other structures of SARS-CoV $\mathrm{M}^{\text {pro }}$ reported previously. For example, the r.m.s.d. between equivalent $\mathrm{C}^{\alpha}$ atoms of this dimeric structure and the structure of wild-type $\mathrm{M}^{\text {pro }}$ at $\mathrm{pH} 8.0$ is $0.59 \AA$ (Yang et al., 2003). The r.m.s.d. is $0.41 \AA$ when the structure is compared with that of wild-type $\mathrm{M}^{\text {pro }}$ in complex with a peptide aldehyde inhibitor (Zhu et al., 2011). However, the position of domain III is different when these structures are compared (see below) and has been excluded from the comparisons.

Our crystallographic analysis of the R298A mutant shows that there is no evidence for any electron density beyond the $\mathrm{C}^{\beta}$ atom of this side chain (Fig. $2 c$ ). This is a confirmation that our protein carries the R298A mutation and that only mutant protein was crystallized in our experiments. Unfortunately, we cannot observe the complete electron density of the peptide substrate, even though there is some residual electron density

\footnotetext{
${ }^{\mathbf{1}}$ Supplementary material has been deposited in the IUCr electronic archive (Reference: DW5035). Services for accessing this material are described at the back of the journal.
}

near the His41/Cys145 catalytic dyad of subunit $A$ (green mesh in Fig. $2 d$ ). To evaluate the possible substrate-binding mode in this structure, we generated a model with the P3-P1 substrate residues (Val-Leu-Gln) based on the structure of the C145A mutant (Hsu, Kuo et al., 2005). In the model, the side chains of P1 Gln and P2 Leu are able to fill the residual electron density and an $\mathrm{O}$ atom of the carboxyl group of P1 Gln is located in the oxyanion hole (Fig. $2 d$ ). In contrast, in subunit $B$ there is no residual electron density at the same position, suggesting that the statuses of the two active sites in the dimer may not be identical, although the amide $\mathrm{N}$ atoms of Gly143 and Cys 145 in each subunit are oriented into the oxyanion hole. In addition, most of the residues in the active site, including Phe140, His163, Glu166, His172 and the catalytic dyad His41/Cys145, are in the same positions as those of wild-type $\mathrm{M}^{\text {pro }}$ (Yang et al., 2003). This seems to confirm that the catalytic machinery of the R298A dimer is functional.

\subsection{Comparison with the monomeric structure of the R298A mutant}

The mutual arrangement of the domains of the R298A mutant is changed dramatically and therefore we compared our dimer structure with that of the monomer (PDB entry 2qcy; Shi et al., 2008) based on superposition of domains I and II. As shown in Fig. 3(a), the most obvious conformational change is a $33^{\circ}$ rotation of domain III. This rotation allows steric hindrance between the two copies of domain III during dimerization to be avoided. The variability of the mutual domain arrangement suggests that conversion from the monomeric form to the dimeric form is feasible. Nevertheless, the dimerization of some other monomeric mutations, such as G11A and S139A, still needs to be confirmed, although they show a similar overall structure to the R298A monomer (Chen et al., 2008; Hu et al., 2009).

The structural comparison also shows that in the present structure the short $3{ }_{10}$-helix of residues Ser139-Phe140Leu141 is disrupted and adopts a loop conformation similar to that of the wild type (Fig. $3 b$; see below). The unfolding of this helix enables the insertion of the $\mathrm{N}$-finger of the other subunit, which is further stabilized by the interaction of Glu166 with Ser1' (primed residue numbers indicate subunit $B$; Fig. $3 b$ ). Most importantly, the key stacking interaction between the rings of Phe140 and His163, which is important to prevent His163 from being protonated (Yang et al., 2003), are maintained (Fig. 2d). This ensures that His163 can efficiently interact with the side chain of P1 Gln. In contrast to the collapsed substrate-binding site in the R298A monomer (Shi et al., 2008), there is a large and complete substrate-binding pocket in each subunit (Fig. 3c). This further suggests that both subunits of the R298A dimer are catalytically active.

In addition, at the dimer interface of the present structure, ion pairs and hydrogen bonds, such as Arg4'-Glu290 and Ser139-Gln299', can also be seen, which is a similar situation to that of the wild-type structure (Fig. $3 b$; see below). All of these observations confirm that the dimerization of R298A is very similar to that of wild-type $\mathrm{M}^{\mathrm{pro}}$, with only minor differ- 
ences. Owing to the lack of a monomeric wild-type $\mathrm{M}^{\text {pro }}$ structure, the monomeric and dimeric R298A structures provide valuable insights into the dimerization process of the enzyme, which undergoes a dramatic mutual arrangement of the domains (Hsu, Kuo et al., 2005; Chen et al., 2008, 2010; Cheng et al., 2010; Li et al., 2010).

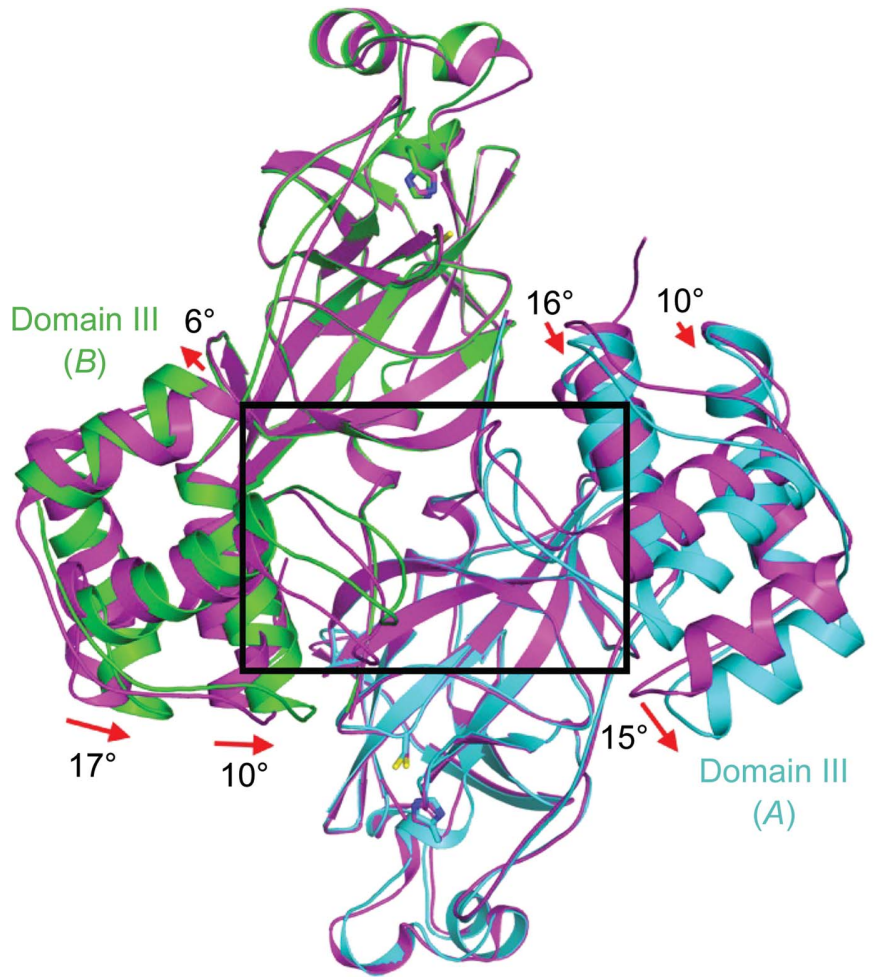

(a)

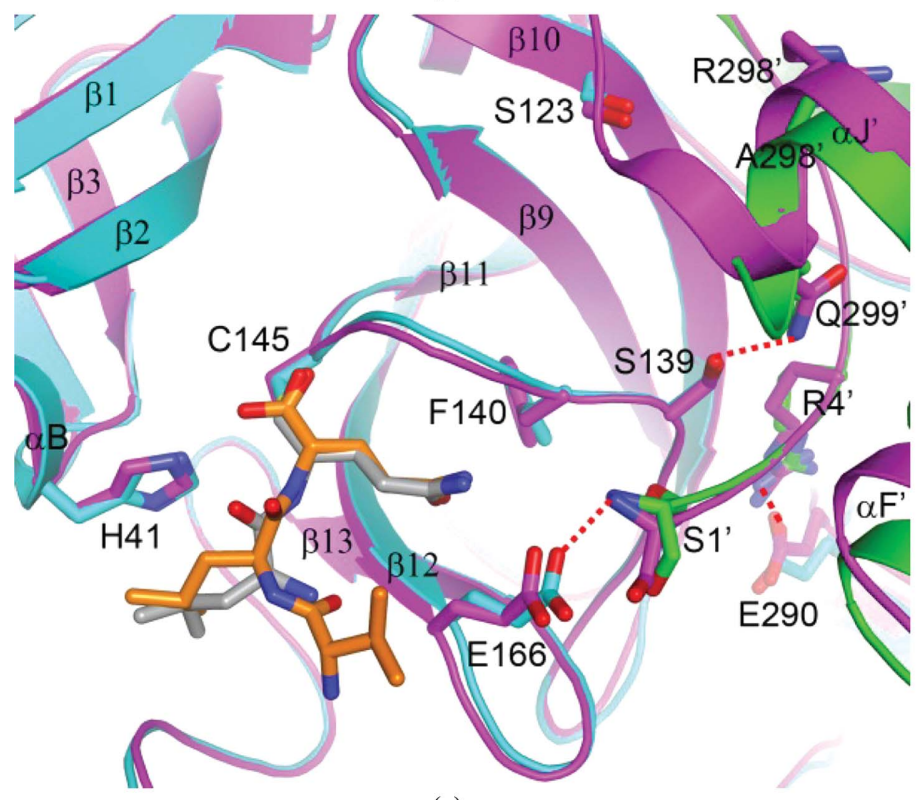

(c)

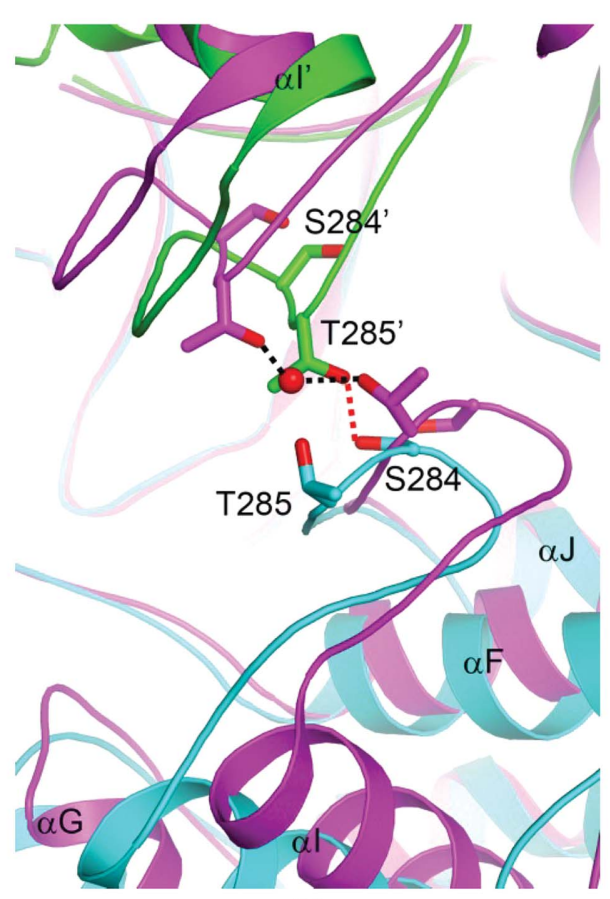

(b)

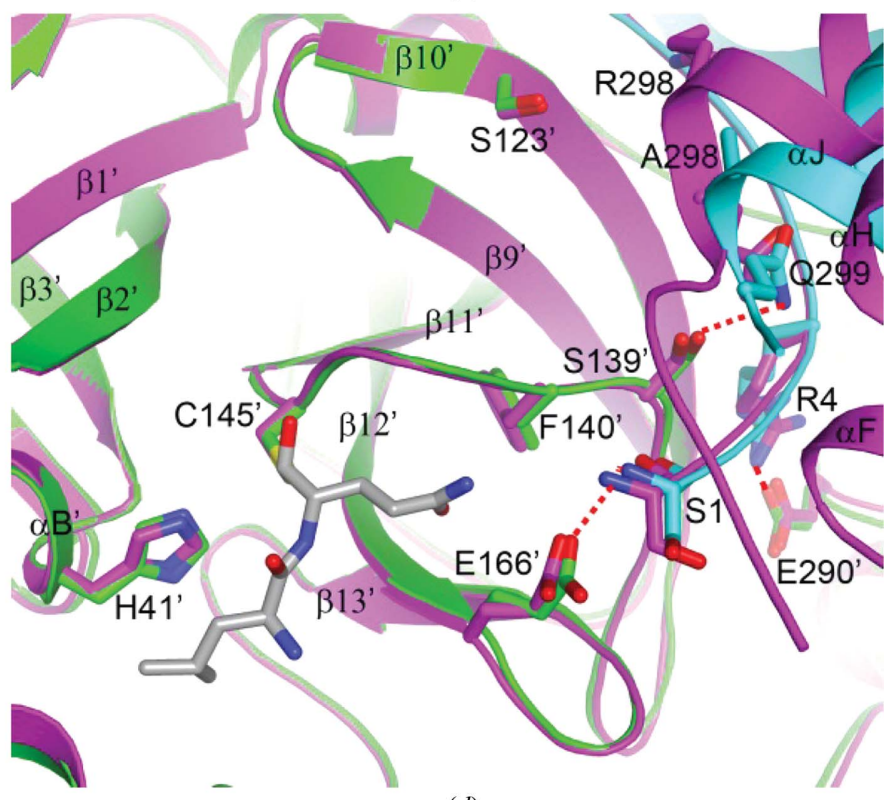

(d)

Figure 4

Comparisons with the structure of wild-type $\mathrm{M}^{\mathrm{pro}}$. (a) An overlay of the current structure of the R298A dimer (cyan and green) with that of wild-type $\mathrm{M}^{\text {pro }}$ in complex with peptidyl aldehyde inhibitor (magenta; PDB entry 3snd; Zhu et al., 2011). The red arrows show the orientation change affecting the two domain IIIs. The region in the box is enlarged in $(b)$. (b) The hydrogen-bonding interaction between the two domain IIIs. The red dashed line indicates the hydrogen bond between Ser284 and Thr285' in the R298A mutant, while the black dashed lines show hydrogen bonds between the two Thr285 residues and the associated water (red sphere) in wild-type $\mathrm{M}^{\text {pro }} .(c)$ and $(d)$ show the detailed interactions of the two protomers near the active sites of subunit $A$ (cyan) and subunit $B$ (green), respectively. The red dashed lines indicate hydrogen bonds and ion pairs for the R298A mutant, while wild-type $\mathrm{M}^{\text {pro }}$ (magenta) shows the same interactions (PDB entry 3snd; Zhu et al., 2011). Overlay of the P2-P1 residues in the two structures (orange in R298A structures and grey in wild-type $\mathrm{M}^{\text {pro }}$, respectively) confirmed that one $\mathrm{O}$ atom of the carboxyl group of P1 Gln was located in the oxyanion hole. 


\subsection{Reorganization of the dimer in the $R 298 \mathrm{~A}$ mutant}

The R298A mutant shows an apparent change in the relative orientation of domain III. When we compared our structure with two wild-type structures (Yang et al., 2003; Zhu et al., 2011), namely the free enzyme at pH 8.0 (PDB entry 1uk2) and the peptide aldehyde inhibitor complex (PDB entry 3 snd), a relative shift of 5-24 could be observed for the two copies of domain III in the dimer compared with the free enzyme, while there was a shift of $6-17^{\circ}$ compared with the inhibitor complex (Fig. 4a). Interestingly, we found that there is a hydrogen-bonding interaction between residues Ser284 and Thr285' in the $\alpha \mathrm{I}-\alpha \mathrm{J}$ loop, while there is a water molecule between the two Thr285 residues in the structure of wild-type $\mathrm{M}^{\text {pro }}$ (Fig. 4b). The direct contact between the two $\alpha \mathrm{I}-\alpha \mathrm{J}$ loops leads to a shift of the $\alpha \mathrm{I}$ and $\alpha \mathrm{J}$ helices, further causing the change in the orientation of the whole domain III. This observation also suggests that the two copies of the folded domain III are able to bind to each other by this interaction at the initial stage and to wait until the swapping of the $\alpha$-helix to form a three-dimensional domain-swapped dimer or a more stable and super-active octameric $\mathbf{M}^{\text {pro }}$ (Zhang et al., 2010; Kang et al., 2012).

Furthermore, in the present structure each domain III is even closer to domains I and II of the other subunit, although subunit $A$ shows a larger change in orientation than subunit $B$. At the dimer interface of the R298A mutant, in addition to two intermolecular Arg4-Glu290 ion pairs (Arg4-Glu290' and Arg4'-Glu290), there are two Ser139...Gln299 hydrogen bonds (Ser139...Gln299' and Ser139'..Gln299) and two Ser1-Glu166 ion pairs (Ser1-Glu166' and Ser1'-Glu166); this contrasts with some of the structures of wild-type $\mathrm{M}^{\text {pro }}$ (PDB entries $1 \mathrm{uk} 2$ and $1 \mathrm{uk} 4)$, which only show one pair of each. A similar assembly can also be observed in the structures of authentic wild-type $\mathrm{M}^{\text {pro }}$ (PDB entry $2 \mathrm{~h} 2 \mathrm{z}$ ) and its complex with a peptide aldehyde inhibitor (PDB entry 3snd), although in these two structures the Arg298 residues do not interact with Ser123 of the other subunit (Figs. $4 c$ and $4 d$; Xue et al., 2007; Zhu et al., 2011). This suggests that $\mathrm{M}^{\text {pro }}$ may not require all of the possible intermolecular interactions for dimerization, especially in the presence of substrates or peptidyl inhibitors. Moreover, previous studies have suggested that Glu166 plays a pivotal role in connecting the substrate-binding site to the dimer interface (Yang et al., 2003; Chen et al., 2008; Cheng et al., 2010). In our structure, the interactions between the mainchain amide of Ser1 with the carboxyl group of the Glu166 side chain of the other subunit provide direct evidence to explain why the mutation at Glu166 blocks substrate-induced dimerization of $\mathrm{M}^{\text {pro }}$ (Cheng et al., 2010). Remarkably, mutation of Arg298, which should be detrimental to dimerization, may be compensated by these interactions, while most of the other residues in the active site show only small changes.

\section{Conclusion}

Our studies show that SARS-CoV $\mathrm{M}^{\text {pro }}$, the dimerization of which is important for its catalytic activity, is able to tolerate large orientation changes, especially involving domain III. Mutation of Arg298, when introduced at the dimer interface, disturbs the dimerization; nevertheless, in the presence of peptide substrate the dimerization is able to be induced or rescued by intermolecular hydrogen-bond (Ser139 . . Gln299) and ion-pair (Ser1-Glu166) interactions. Based on the existence of a complete substrate-binding pocket and a complete oxyanion hole, we suggested that the dimer is still catalytically active, even though there are conformational rearrangements of the two copies of domain III in the dimer. AEC experiments confirmed that the kinetic parameters of the R298A mutant are similar to those of wild-type $\mathrm{M}^{\text {pro }}$. The present studies provide valuable insights into the mechanisms that control the monomer-dimer switch during the maturation process of $\mathrm{M}^{\text {pro }}$.

We thank G.-G. Chang and the reviewers for helpful discussions. This research was supported by grants from the National Health Research Institute, Taiwan (NHRI-EX1019947SI) and the National Science Council, Taiwan (98-2320-B010-026-MY3) to C-YC. Portions of this research were carried out at the National Synchrotron Radiation Research Center, a national user facility supported by the National Science Council of Taiwan, ROC. The Synchrotron Radiation Protein Crystallography Facility is supported by the National Core Facility Program for Biotechnology.

\section{References}

Anand, K., Palm, G. J., Mesters, J. R., Siddell, S. G., Ziebuhr, J. \& Hilgenfeld, R. (2002). EMBO J. 21, 3213-3224.

Anand, K., Ziebuhr, J., Wadhwani, P., Mesters, J. R. \& Hilgenfeld, R. (2003). Science, 300, 1763-1767.

Brown, P. H. \& Schuck, P. (2006). Biophys. J. 90, 4651-4661.

Chen, S., Hu, T., Zhang, J., Chen, J., Chen, K., Ding, J., Jiang, H. \& Shen, X. (2008). J. Biol. Chem. 283, 554-564.

Chen, S., Jonas, F., Shen, C. \& Hilgenfeld, R. (2010). Protein Cell, 1, 59-74.

Cheng, S.-C., Chang, G.-G. \& Chou, C.-Y. (2010). Biophys. J. 98, 1327-1336.

Chou, C.-Y., Chang, H.-C., Hsu, W.-C., Lin, T.-Z., Lin, C.-H. \& Chang, G.-G. (2004). Biochemistry, 43, 14958-14970.

Chou, C.-Y., Chien, C.-H., Han, Y.-S., Prebanda, M. T., Hsieh, H.-P., Turk, B., Chang, G.-G. \& Chen, X. (2008). Biochem. Pharmacol. 75, 1601-1609.

Chou, C.-Y., Hsieh, Y.-H. \& Chang, G.-G. (2011). Methods, 54, 76-82. Chou, C.-Y. \& Tong, L. (2011). J. Biol. Chem. 286, 24417-24425.

Emsley, P. \& Cowtan, K. (2004). Acta Cryst. D60, 2126-2132.

Gorbalenya, A. E., Enjuanes, L., Ziebuhr, J. \& Snijder, E. J. (2006). Virus Res. 117, 17-37.

Hegyi, A. \& Ziebuhr, J. (2002). J. Gen. Virol. 83, 595-599.

Hoek, L. van der, Pyrc, K., Jebbink, M. F., Vermeulen-Oost, W., Berkhout, R. J., Wolthers, K. C., Wertheim-van Dillen, P. M., Kaandorp, J., Spaargaren, J. \& Berkhout, B. (2004). Nature Med. 10, 368-373.

Hsu, M.-F., Kuo, C.-J., Chang, K.-T., Chang, H.-C., Chou, C.-C., Ko, T.-P., Shr, H.-L., Chang, G.-G., Wang, A. H.-J. \& Liang, P.-H. (2005). J. Biol. Chem. 280, 31257-31266.

Hsu, W.-C., Chang, H.-C., Chou, C.-Y., Tsai, P.-J., Lin, P.-I. \& Chang, G.-G. (2005). J. Biol. Chem. 280, 22741-22748.

Hu, T., Zhang, Y., Li, L., Wang, K., Chen, S., Chen, J., Ding, J., Jiang, H. \& Shen, X. (2009). Virology, 388, 324-334.

Kang, X., Zhong, N., Zou, P., Zhang, S., Jin, C. \& Xia, B. (2012). Proc. Natl Acad. Sci. USA, 109, 14900-14905. 
Lau, S. K. P., Woo, P. C. Y., Li, K. S. M., Huang, Y., Tsoi, H.-W., Wong, B. H. L., Wong, S. S. Y., Leung, S.-Y., Chan, K.-H. \& Yuen, K.-Y. (2005). Proc. Natl Acad. Sci. USA, 102, 14040-14045.

Li, C., Qi, Y., Teng, X., Yang, Z., Wei, P., Zhang, C., Tan, L., Zhou, L., Liu, Y. \& Lai, L. (2010). J. Biol. Chem. 285, 28134-28140.

Li, W. et al. (2005). Science, 310, 676-679.

Lin, P.-Y., Chou, C.-Y., Chang, H.-C., Hsu, W.-C. \& Chang, G.-G. (2008). Arch. Biochem. Biophys. 472, 34-42.

McCoy, A. J., Grosse-Kunstleve, R. W., Adams, P. D., Winn, M. D., Storoni, L. C. \& Read, R. J. (2007). J. Appl. Cryst. 40, 658-674.

Murshudov, G. N., Skubák, P., Lebedev, A. A., Pannu, N. S., Steiner, R. A., Nicholls, R. A., Winn, M. D., Long, F. \& Vagin, A. A. (2011). Acta Cryst. D67, 355-367.

Otwinowski, Z. \& Minor, W. (1997). Methods Enzymol. 276, 307-326.

Schuck, P. (2000). Biophys. J. 78, 1606-1619.

Shi, J., Sivaraman, J. \& Song, J. (2008). J. Virol. 82, 4620-4629.

Snijder, E. J., Bredenbeek, P. J., Dobbe, J. C., Thiel, V., Ziebuhr, J., Poon, L. L. M., Guan, Y., Rozanov, M., Spaan, W. J. M. \& Gorbalenya, A. E. (2003). J. Mol. Biol. 331, 991-1004.

Weiss, S. R. \& Navas-Martin, S. (2005). Microbiol. Mol. Biol. Rev. 69, 635-664.
Woo, P. C. Y., Lau, S. K. P., Chu, C.-M., Chan, K. H., Tsoi, H. W., Huang, Y., Wong, B. H. L., Poon, R. W. S., Cai, J. J., Luk, W.-K., Poon, L. L. M., Wong, S. S. Y., Guan, Y., Peiris, J. S. M. \& Yuen, K.-Y. (2005). J. Virol. 79, 884-895.

Wu, C.-Y., King, K.-Y., Kuo, C.-J., Fang, J.-M., Wu, Y.-T., Ho, M.-Y., Liao, C.-L., Shie, J.-J., Liang, P.-H. \& Wong, C.-H. (2006). Chem. Biol. 13, 261-268.

Xue, X., Yang, H., Shen, W., Zhao, Q., Li, J., Yang, K., Chen, C., Jin, Y., Bartlam, M. \& Rao, Z. (2007). J. Mol. Biol. 366, 965-975.

Xue, X., Yu, H., Yang, H., Xue, F., Wu, Z., Shen, W., Li, J., Zhou, Z., Ding, Y., Zhao, Q., Zhang, X. C., Liao, M., Bartlam, M. \& Rao, Z. (2008). J. Virol. 82, 2515-2527.

Yang, H., Yang, M., Ding, Y., Liu, Y., Lou, Z., Zhou, Z., Sun, L., Mo, L., Ye, S., Pang, H., Gao, G. F., Anand, K., Bartlam, M., Hilgenfeld, R. \& Rao, Z. (2003). Proc. Natl Acad. Sci. USA, 100, 13190-13195.

Zhang, S., Zhong, N., Xue, F., Kang, X., Ren, X., Chen, J., Jin, C., Lou, Z. \& Xia, B. (2010). Protein Cell, 1, 371-383.

Zhao, Q., Li, S., Xue, F., Zou, Y., Chen, C., Bartlam, M. \& Rao, Z. (2008). J. Virol. 82, 8647-8655.

Zhu, L., George, S., Schmidt, M. F., Al-Gharabli, S. I., Rademann, J. \& Hilgenfeld, R. (2011). Antiviral Res. 92, 204-212. 